\title{
PF4V1 affects the progression of oral squamous cell carcinoma by regulating Wnt/ $\beta$-catenin pathway and angiogenesis
}

\author{
Cuiping $\mathrm{Li}^{1 \dagger}$, Weidong Jiang ${ }^{2 \dagger}$, Yang Zhou ${ }^{3 \dagger}$, Xuanping Huang ${ }^{2}$ and Nuo Zhou ${ }^{1,2^{*}}$ (1)
}

\begin{abstract}
Platelet factor-4 variant 1 (PF4V1) was recently described as a natural non-allelic gene variant of platelet factor-4 (PF4), which has been closely associated with the growth and metastasis of various cancers. Our previous research showed that PF4V1 was related to oral squamous cells carcinoma (OSCC) metastasis. Howerver, it is still not clear about the functional role of PF4V1 in OSCC. In this study, stably transfected cell lines were constructed and the expression level of PF4V1 was verified by real-time polymerase chain reaction (RT-PCR) and western blot. The effect of PF4V1 on proliferation, migration, invasion, and apoptosis of oral cancer $(\mathrm{OC})$ cells were detected. Moreover, a xenograft tumor model was constructed to evaluate the effect of PF4V1 on OSCC in vivo. Indicators of Wnt/ $\beta$-catenin, angiogenesis and epithelial-mesenchymal transition (EMT) pathways were also examined. Stable cell lines with overexpression and inhibited expression of PF4V1 were constructed successfully. After stable transfection, PF4V1 significantly promoted the proliferation, migration, and invasion of OC cells in vitro, and their tumor formation in vivo. Furthermore, PF4V1 remarkably promoted the expression of $\beta$-catenin, VEGF, and FGF but suppressed the expression of GSK-3 $\beta$. There was no statistically significant correlation between PF4V1 and EMT pathway. This study provides evidence that PF4V1 promotes the proliferation, migration, invasion and tumor formation of OC cells by regulating the Wnt/ $\beta$-catenin pathway and angiogenesis. Our findings suggest that PF4V1 could be a very promising target of OSCC therapy in the future.
\end{abstract}

Keywords: PF4V1, Oral squamous cells carcinoma, Wnt/ß-catenin, Angiogenesis

\section{Introduction}

Oral squamous cells carcinoma (OSCC) is the 3rd and 6th most common cancer type in developing countries and worldwide, respectively [1]. Although there are corresponding diagnostic and treatment strategies in clinics for the prevention and treatment of OSCC, unfavourable prognosis and low survival rate remain a problem for OSCC patients [2]. The appearance of migrated feature

\footnotetext{
*Correspondence: gxzhounuo@sina.cn

${ }^{\dagger}$ Cuiping Li, Weidong Jiang and Yang Zhou have contributed equally to this work

${ }^{2}$ Department of Oral and Maxillofacial Surgery, The Affiliated Stomatology Hospital of Guangxi Medical University, Nanning 530021, People's Republic of China

Full list of author information is available at the end of the article
}

led to the poor prognosis. There is an urgent need to explore the molecular mechanism of tumor formation in OSCC and identify effective bioindicators and therapeutic targets in order to improve the prognosis and treatment efficacy of OSCC and to reduce patient mortality. In recent years, gene therapy has become an increasingly popular focus for a lot of researchers and has currently been used in a wide range of diseases [3, 4], and tumor is no exception [5]. However, there are few investigations for the use of gene therapy in OSCC. Thus, the molecular mechanism underlying OSCC development remains unclear.

Platelet factor-4 (PF4) was described as the first chemokine that inhibited neovascularization [6]. As the product of the nonallelic variant gene of PF4, platelet 
factor-4 variant 1(PF4V1) was isolated initially from thrombin-stimulated human platelets and purified to homogeneity [6]. Although the difference between secreted PF4 and PF4V1 was merely three amino acids, PF4V1 had a greater potential than PF4 in inhibiting chemotaxis of human microvascular endothelial cells. Compared with PF4 in vivo, PF4V1 was found to be more effective in suppressing bFGF-induced angiogenesis [6]. Thus, PF4V1 released from activated platelets influenced angiogenesis and vascular diseases. Up to now, PF4 has been a hot research topic as a potentially powerful antitumor agent. A previous study confirmed that recombinant PF4V1 was a stronger angiostatin chemokine in inhibiting tumor growth and metastasis than PF4 in a variety of different solid tumor types [7]. Our study explored the potential of PF4V1 as an anti-tumor agent in OSCC.

As one of the classic pathways, the Wnt/ $/$-catenin signaling pathway participates in cell signal transduction, and abnormality of Wnt signaling has become the foundation of a large number of congenital disorders and carcinomas [8, 9]. Furthermore, Wnt signaling played a key role in the regulation of the fate, proliferation, migration and survival of oral cancer (OC) cells [10]. Beyond that, epithelial-mesenchymal transition (EMT) has been verified to expedite the conversion of epithelial cells to mesenchymal cells [11], which is also one of the key factors in OSCC initiation and progression [12-14]. A report showed that inhibited EMT reduced the migration and invasion of $\mathrm{OC}$ cells by suppressing the activity of $\mathrm{Wnt} / \beta$-catenin signaling pathway, thereby achieving the objective of inhibiting cell growth and promoting cell apoptosis [15]. More and more researches have revealed that PF4V1 regulated cell differentiation, proliferation, and apoptosis $[7,16,17]$. Our previous study showed that PF4V1 had a close relationship with OSCC [18]. Nevertheless, there has been no study that reports the relationship between PF4V1 and Wnt/ $\beta$-catenin and EMT pathways in OSCC. To provide evidence for this potentially powerful anti-OSCC agent, our study explored how PF4V1 affected the proliferation, migration and invasion of OSCC through different signaling pathways.

\section{Materials and methods \\ Cell transfection}

CAL-27 and SCC-9 cells (Shanghai Institute of Cell Biology, Chinese Academy of Sciences) were cultured in $90 \%$ dulbecco's modified Eagle's medium (DMEM) (Gibco BRL, Carlsbad, CA, USA) supplemented with $10 \%$ fetal bovine serum (FBS) (Gibco BRL, Carlsbad, CA, USA). The pLV-EGFP-PF4V1, pLV-EGFP-shRNAPF4V1 and the control vectors were commercially synthesized by Cyagen Biosciences (Shanghai, China). The
pLV-EGFP-shRNA-PF4V1 lentiviral vectors and their corresponding control vector were applied to decrease the expression level of PF4V1. The pLV-EGFP-PF4V1 lentiviral vector and its corresponding control vector were applied to increase the expression level of PF4V1. The three candidate target shRNA sequences were GAG CTGAAGCTGAAGAAGATG (shRNA\#1), GTGAAA GAATCTTCTGATGTT (shRNA\#2), and GCTGAC ACATCACAATTTCAT (shRNA\#3). Then, the cells were transfected according to the manufacturer's protocol. Preliminary tests of two cell lines were carried out to determine the multiplicity of infection (MOI) value of lentivirus and the MOI gradient was $2.5,5,10,20,40$. Cells were placed in a 24 -well plate with $1 \times 10^{5}$ cells per well before lentivirus transfection and the number of cells transfected with lentivirus were about $2 \times 10^{5} /$ well. After $24 \mathrm{~h}$, the original medium was replaced with $2 \mathrm{ml}$ fresh medium containing $6 \mu \mathrm{g} / \mathrm{ml}$ polybrene, and then an appropriate amount of virus suspension was added into the medium. Fresh medium replaced the viral medium after $24 \mathrm{~h}$ of incubation at $37^{\circ} \mathrm{C}$. The antibiotic-resistant transfected cells were selected and enriched by applying different concentrations of puromycin ( 7 days with $2 \mu \mathrm{g} / \mathrm{ml}$ puromycin and 3 days with $0.5 \mu \mathrm{g} / \mathrm{ml}$ puromycin). The expression level of PF4V1 was verified by RTPCR and western blot at 10 days post-transfection. Total RNA was extracted from the cell lines using TRIzol reagent (Ambion, Life Technologies, USA) and was reversely transcribed into cDNA using the manufacturer's specifications of commercially available kits (Takara). All primers were shown in Table 1. Relative quantification of mRNA expression was performed using the SYBR Green RT-PCR Kit (Takara) normalized to that of GAPDH in an ABI StepOne/StepOnePlus Detection System (Applied Biosystems) with the following reaction conditions: stage 1: $95^{\circ} \mathrm{C} / 30 \mathrm{~s}$ for 1 cycle; stage $2: 95^{\circ} \mathrm{C} / 5 \mathrm{~s}$, and $60^{\circ} \mathrm{C} / 30 \mathrm{~s}$ for 40 cycles; stage $3: 95^{\circ} \mathrm{C} / 15 \mathrm{~s}, 60^{\circ} \mathrm{C} / 60 \mathrm{~s}$ and $95^{\circ} \mathrm{C} / 15 \mathrm{~s}$ for 1 cycle. The results were analyzed using the $2^{-\Delta \Delta \mathrm{Ct}}$ method.

Total protein was extracted from the cell lines. Cells were lysed using RIPA buffer with a proteinase inhibitor (Beyotime Biotechnology). The concentration of total protein was detected by utilizing BCA assay kits. The loading quantity of western blotting test was $50 \mu \mathrm{g}$ to detect the expression level of PF4V1protein. The antibody information was as follows: rabbit anti-PF4V1 (1:1000 for western blot, Sigma), mouse anti-GAPDH (1:2000, Sino Biological Inc.). The secondary antibody was anti-rabbit/mouse IgG (1:2000, Sino Biological Inc.). The protein bands were visualized by using a chemiluminescence reagent kit (Thermo Scientific, USA). 
Table 1 The primer information in RT-PCR assay

\begin{tabular}{|c|c|c|c|}
\hline Pathway & Gene name & Forward primer $\left(5^{\prime}-3^{\prime}\right)$ & Reverse primer $\left(5^{\prime}-3^{\prime}\right)$ \\
\hline \multirow[t]{6}{*}{ Wnt/B-catenin pathway } & PF4V1 & CAGAACCCCAGCCCGACTTTCCC & TTAATCAGCAGATTCATCACCTGCC \\
\hline & GAPDH & СTCCTCCACCTTTGACGCTG & TCCTCTTGTGCTCTTGCTGG \\
\hline & VEGF & GCCTTGCCTTGCTGCTCTACC & GGTCTCGATTGGATGGCAGTAGC \\
\hline & GSK-3 $\beta$ & CAGAGACAAGGACGGCAGCAAG & GGCGACCAGTTCTCCTGAATCAC \\
\hline & FGF & GTGCTAACCGTTACCTGGCT & CCCAGTTCGTTTCAGTGCCA \\
\hline & $\beta$-catenin & GGCTCTTGTGCGTACTGTCCTTC & GCTTCTTGGTGTCGGCTGGTC \\
\hline \multirow[t]{3}{*}{ EMT pathway } & Snail1 & GGCTCCTTCGTCCTTCTCCTCTAC & CCAGGCTGAGGTATTCCTTGTTGC \\
\hline & Snail2 & CCATGCCTGTCATACCACAACCAG & GGAGGAGGTGTCAGATGGAGGAG \\
\hline & Twist & CACCATCCTCACACCTCTGCATTC & GGCTGATTGGCACGACCTCTTG \\
\hline \multirow[t]{2}{*}{ Apoptosis-related genes } & $\mathrm{BCl}-2$ & ATCGCCCTGTGGATGACTGAG & CAGCCAGGAGAAATCAAACAGAGG \\
\hline & Caspase-3 & CCTGTGGCTGTGTATCCGTG & CCGAGATGTCATTCCAGTGC \\
\hline
\end{tabular}

\section{Colony formation and cell proliferation assays}

OC cells were seeded into 6-well culture plates with 500 cells/well for 15 days to evaluate the cell growth. Colonies were fixed with $4 \%$ paraformaldehyde for $15 \mathrm{~min}$ and then stained with $0.5 \%$ crystal violet for $10 \mathrm{~min}$. The images of colony formation were examined under an olympus microscope.

For the viability assays, cells were seeded into 96-well culture plates with 4000 cells/well. pLV-EGFP-PF4V1 overexpression vector, PF4V1-shRNA interfering vector, as well as their corresponding control vectors CtrlPF4V1 and Ctrl-shRNA were transfected into CAL-27 and SCC-9 cells, respectively. Cell viability was examined using the Cell Counting Kit-8 (CCK-8) (Dojindo, Tokyo, Japan) on the basis of the manufacturer's procedures at $24 \mathrm{~h}, 48 \mathrm{~h}, 72 \mathrm{~h}$ incubation time points. Ten $\mu \mathrm{l} \mathrm{CCK-} 8$ solution was added to the cells and then incubated in the dark for about $4 \mathrm{~h}$. The absorbance at $450 \mathrm{~nm}$ was measured by the Microplate Reader (Thermo Scientific ${ }^{\text {TM }}$ Multiskan $^{\mathrm{TM}}$ GO, Rockford, IL, USA).

\section{Transwell test}

Transwell chambers were coated with either $100 \mu \mathrm{l}$ matrigel (BD Biosciences, Franklin Lakes, New Jersey) to test cell invasion or no matrigel to test cell migration. Each transwell chamber was inoculated with $1 \times 10^{5}$ cells, which were suspended in $100 \mu \mathrm{l}$ serum-free DMEM (Gibco Company). The lower chamber was filled with $800 \mu \mathrm{l}$ complete medium. The cells in the upper chamber were discarded after $48 \mathrm{~h}$ incubation. Then cells in lower chamber were fixed for $15 \mathrm{~min}$ by $4 \%$ paraformaldehyde and stained for $10 \mathrm{~min}$ by crystal violet. Five fields of view were chosen at random for photograph and calculation in the microscope. The cells adhering to the side in the lower chamber were the target for counting, which were invasive or migrating cells.

\section{Cell apoptosis analyzed by flow cytometry and RT-PCR}

The apoptosis rates of CAL-27 and SCC-9 cells after transfection were detected using flow cytometry. The apoptotic assay was conducted at $48 \mathrm{~h}$ after transfection. After collection and washing with cold phosphatebuffered saline (PBS), the cells were stained with Annexin $\mathrm{V}$-APC and propidium iodide (PI) (Keygen Biotech, China) for $15 \mathrm{~min}$ in the dark based on the manufacturer's protocol and were then analyzed by a flow cytometer (Beckman Coulter, Atlanta, USA). The single positive cells of Annexin V-APC were the early apoptotic cells. Double positive cells of Annexin V-APC and PI were necrotic or late apoptotic cells.

The expression of apoptosis-related genes $\mathrm{Bcl}-2$ and Caspase- 3 were detected by RT-PCR. The primer information of Bcl-2 and Caspase-3 was shown in Table 1.

\section{Tumor formation assay in vivo}

The in vivo protocol complied with the relevant regulations of Guangxi Medical University's Animal Ethics Committee. Female nude mice were provided by Beijing Vital River Laboratory Animal Technology Co., Ltd. (Beijing, China). CAL-27 and SCC-9 cells were stably transfected by the empty vector, pLV-EGFP-shRNA-PF4V1 lentiviral vector and $\mathrm{pLV-EGFP-PF4V1} \mathrm{lentiviral} \mathrm{vector.}$ The hypodermis of each mouse was injected with $0.2 \mathrm{ml}$ fresh DMEM (containing $4 \times 10^{5}$ cells). Important indices were tested every 5 days, including the size and weight of tumors, as well as the weight of mice to test the effect of PF4V1 on OSCC in vivo. Tumor tissues were examined with hematoxylin-eosin ( $\mathrm{H} \& \mathrm{E})$ staining to test the effect of PF4V1 on tumor angiogenesis in vivo. 
Analysis of related proteins of Wnt/ $\beta$-catenin pathway, EMT pathway and angiogenesis

Total RNA was extracted and then RT-PCR was conducted. The information of the primers was shown in Table 1. Western blot was used to detect the related proteins of $\mathrm{Wnt} / \beta$-catenin and EMT pathways. BCA protein assay kits detected the total protein concentration. Forty to $60 \mu \mathrm{g}$ of protein was used in western blotting test. The concentration of SDS-PAGE gel was $12 \%$. Membranes were blocked and then incubated overnight with rabbit anti-FGF (1:1000, ARP42005 P050, AVIVA SYSTEMS BIOLOGY), rabbit anti-VEGF (1:1000, MA1-16629, Thermo Fisher Scientific), rabbit anti$\beta$-catenin (1:1000, PA5-19469, Thermo Fisher Scientific), mouse anti-GSK-3 $\beta$ (1:1000, bs-0023 M, BIOSS), anti-snail+slug (1:100, ab180714, Abcam), anti-twist
(1:100, ab50581, Abcam), or rabbit polyclonal anti- $\beta$ actin antibody (1:1000, Cell Signaling Technology, Danvers, USA). The secondary antibody was anti-rabbit IgG (1:2000, Sino Biological Inc.). A chemiluminescent gel imaging system (FluorChem HD2; ProteinSimple; USA) scanned the bands.

\section{Results}

Stably transfected cell lines were constructed successfully The results of preliminary tests showed that MOI value of lentivirus transfection was 10 . The results from RTPCR and western blot showed that the overexpression and suppressed expression of PF4V1 was fulfilled in SCC-9 and CAL-27 cells (Fig. 1). Furthermore, the

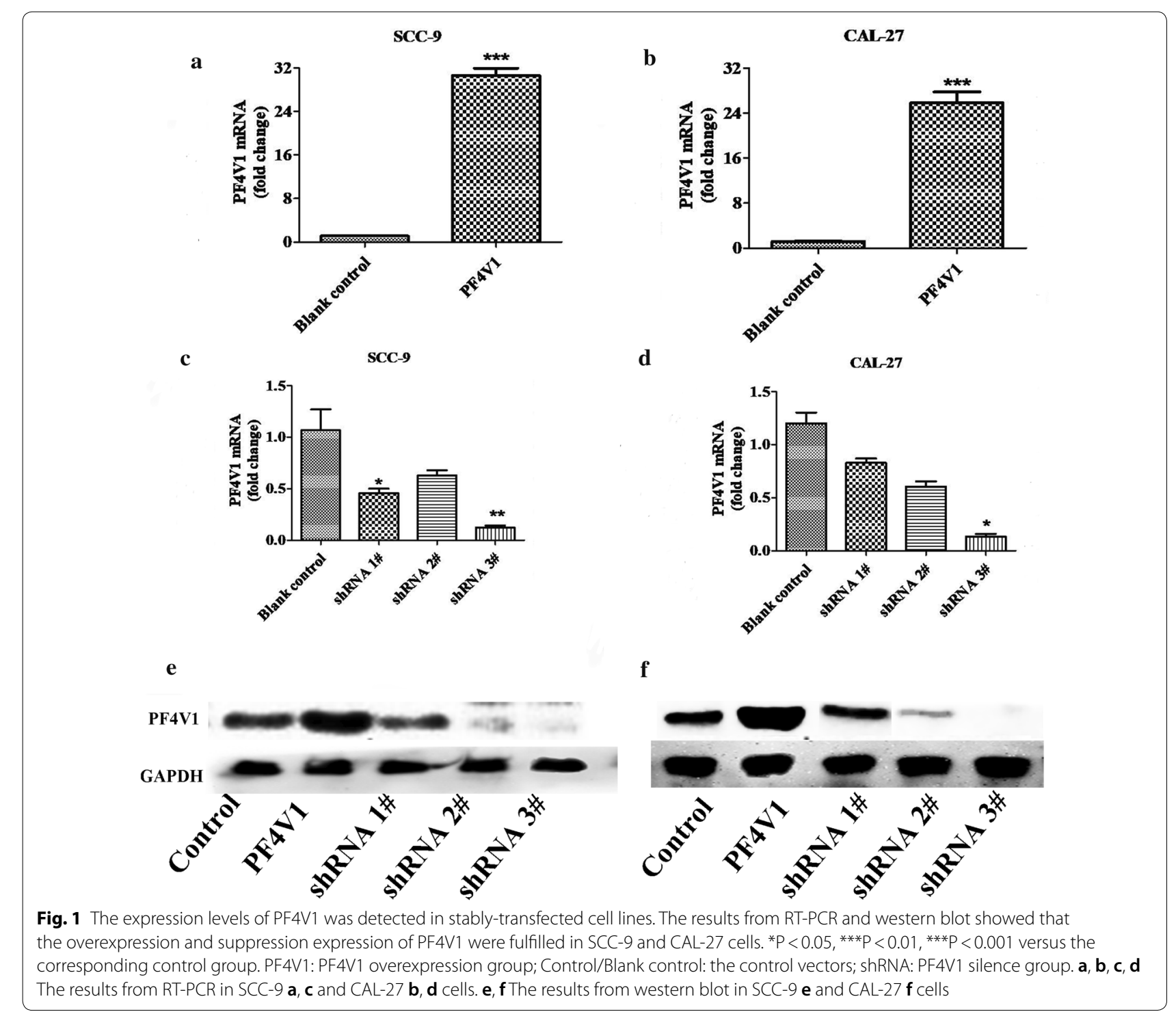


candidate target shRNA 3\# sequence led to the highest transfection effect (Fig. 1).

\section{PF4V1 overexpression promoted the proliferation of OC cells}

The CCK- 8 results showed that PF4V1 overexpression significantly increased the proliferation of the SCC-9 (Fig. 2c) and CAL-27 (Fig. 2g) cells after incubation for $72 \mathrm{~h}$. However, silent expression of PF4V1 inhibited the proliferation of the SCC-9 (Fig. 2b, c) and CAL-27 (Fig. 2e, g) cells when compared with the control group after incubation for $48 \mathrm{~h}$ and $72 \mathrm{~h}(\mathrm{P}<0.05)$. A significant increase of the proliferation of CAL-27 (Fig. 2f) cell was found in PF4V1 overexpression group after incubation for $48 \mathrm{~h}$, but not in SCC-9 cell (Fig. 2b). Moreover, No statistical changes of the proliferation of the SCC-9 (Fig. 2a) and CAL-27 (Fig. 2e) cells were found after incubation for $24 \mathrm{~h}(\mathrm{P}>0.05)$.

Similarly, the cloning formation capabilities of CAL27 (Fig. 2h) and SCC-9 (Fig. 2d) cells enhanced sharply in PF4V1 overexpression group; on the contrary, the cloning efficiency declined in inhibitory expression group.

\section{PF4V1 silent-expression increased the apoptosis of OC cells}

The over-expression of PF4V1 was shown to have significantly decreased the apoptosis rate of OC cells while the silent-expression of PF4V1 had significantly increased the apoptosis rate of $\mathrm{OC}$ cells when compared with the empty vector (Fig. 3a, b). The percentage of live cells was significantly decreased in PF4V1 silencing group (CAL27: $63.73 \%$, SCC-9: $56.89 \%$ ) than that in the control group (CAL-27: 94.61\%, SCC-9: 94.30\%). The percentage of dead cells was significantly decreased in PF4V1 overexpression group (CAL-27: 0.76\%, SCC-9: 1.51\%) than that in the control group (CAL-27: 3.85\%, SCC-9: $4.11 \%)$. Nevertheless, the percentage of dead cells was significantly increased in PF4V1 silencing group (CAL-27: $12.45 \%$, SCC-9: $21.38 \%$ ) than that in the control group (CAL-27: 2.22\%, SCC-9: 2.74\%). The percentage of apoptotic cells was significantly increased in PF4V1 silencing group (CAL-27: 23.82\%, SCC-9: 21.74\%) than that in the control group (CAL-27: 3.17\%, SCC-9: 2.96\%).

The expression level of Caspase-3 decreased significantly in PF4V1 overexpression group (fold decrease: 0.31 for CAL-27, 0.23 for SCC-9) and increased significantly in PF4V1 silencing group compared with the empty vector group (fold increase: 2.8 for CAL-27, 4.74 for SCC-9) (Fig. 3c, d). On the contrary, the expression level of Bcl-2 increased significantly in PF4V1 overexpression group (fold increase: 5.26 for CAL-27, 5.48 for
SCC-9) and decreased significantly in PF4V1 silencing group (fold decrease: 0.28 for CAL-27, 0.32 for SCC-9) (Fig. 3e, f).

\section{PF4V1 overexpression promoted the migration and invasion of $O C$ cells}

As seen in Fig. 4, the results of migration test showed that the number of migration and invasion of OC cells reduced in the PF4V1 silencing group and elevated in the PF4V1 overexpression group compared with the corresponding blank groups $(\mathrm{P}<0.05)$. These findings revealed that PF4V1 overexpression increased the invasion and migration of $\mathrm{OC}$ cells and the downregulation of PF4V1 expression inhibited the invasion and migration of $\mathrm{OC}$ cells. The findings revealed that PF4V1 could affect the migration and invasion of $\mathrm{OC}$ cells.

\section{PF4V1 affected tumor formation of OC cells in vivo}

To examine the effect of PF4V1 on tumor formation, the silencing expression cells, overexpression cells and the control cells were then injected subcutaneously into nude mice. The results demonstrated that the xenograft tumors of PF4V1-knockdown group developed much more quickly, larger and heavier than those in the control group within the first 15 days and then they began to develop much slower, smaller and lighter after 15 days (Fig. 5a, c, $\mathrm{P}<0.001$ ). Conversely, the xenograft tumors of PF4V1-overexpression group developed much more slowly, were smaller and lighter than those in the control group within the first 15 days and they started to develop much more quickly, larger and heavier after 15 days (Fig. 5a, c, $\mathrm{P}<0.001$ ). These results showed that PF4V1 suppressed tumor formation of OC cells in the first 15 days and promoted the tumor formation of $\mathrm{OC}$ cells in vivo after 15 days. The weight of nude mice was lighter in PF4V1-overexpression and PF4V1-knockdown group than those in the corresponding control groups at the last observation time point (Fig. 5b, $\mathrm{P}<0.001$ ). The results of $H \& E$ staining showed increased angiogenesis significantly in PF4V1-overexpression group compared with other controls (Fig. 5e). Moreover, most of tumor tissues were highly differentiated in PF4V1-knockdown group and less differentiated in PF4V1-overexpressing group (Fig. 5e).

\section{PF4V1 affected the activity of Wnt/ $\beta$-catenin signaling pathway and angiogenesis}

The results showed (Fig. 6) that the expression levels of $\beta$-catenin, VEGF, and FGF in OC cells with PF4V1 overexpression were significantly higher than that in the corresponding control group, nevertheless the expression level of GSK-3 $\beta$ in those OC cells was significantly lower than that in the corresponding control group. 

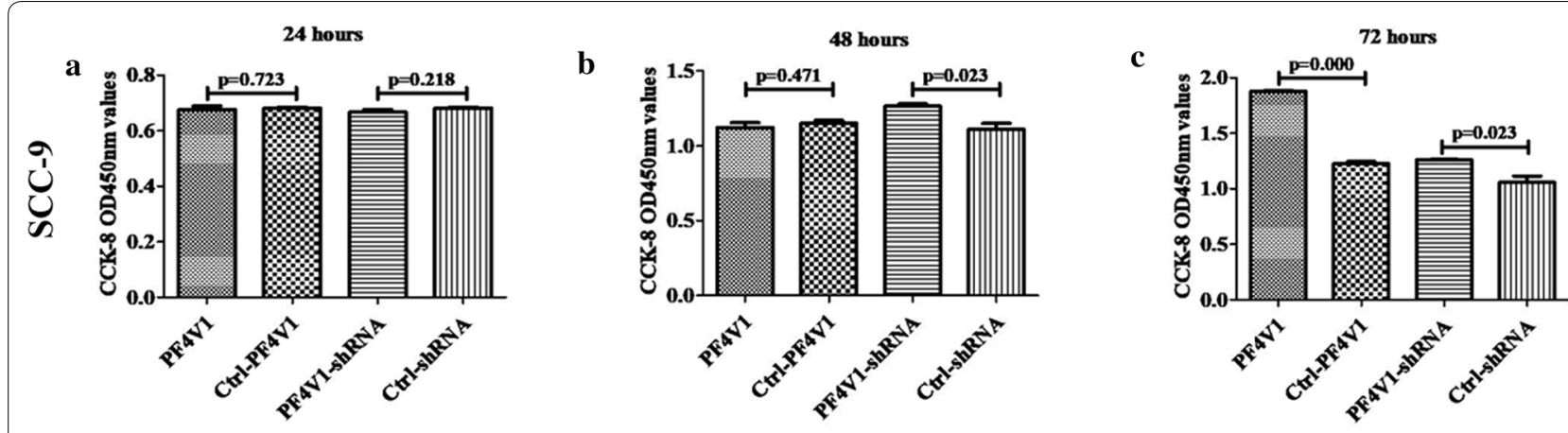

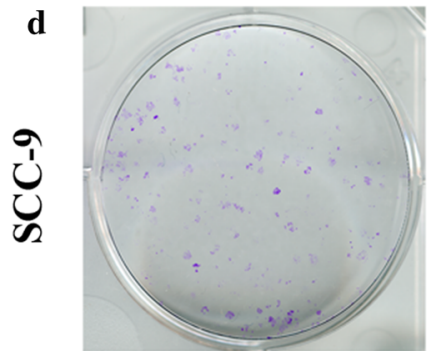

PF4V1

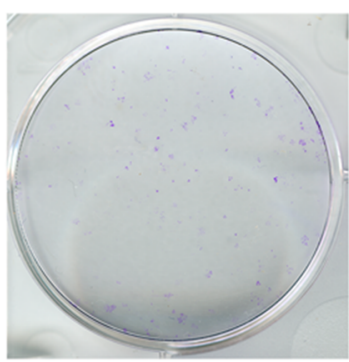

Ctrl-PF4V1

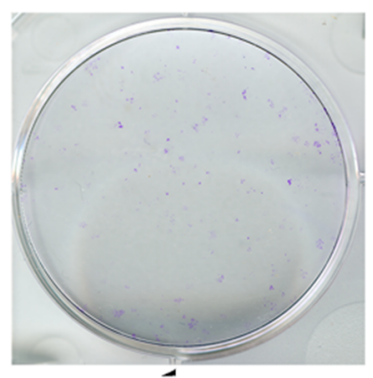

PF4V1-shRNA

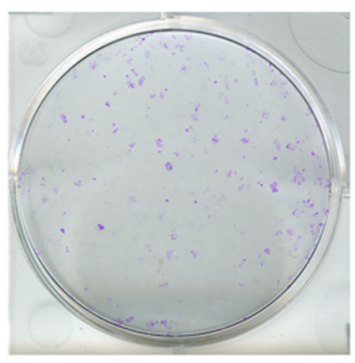

Ctrl-shRNA
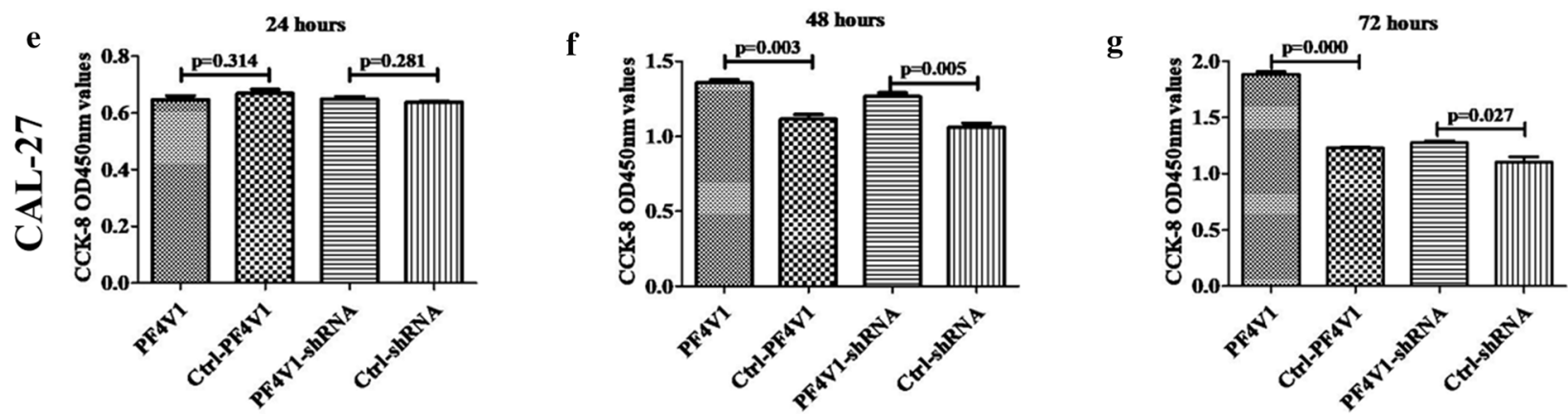

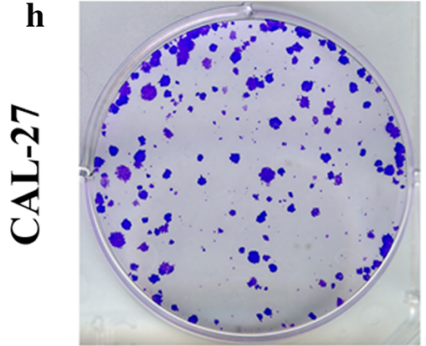

PF4V1

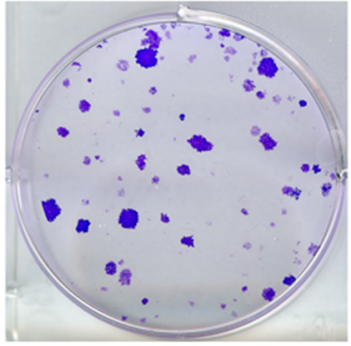

Ctrl-PF4V1

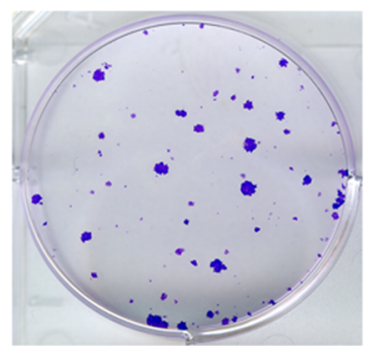

PF4V1-shRNA

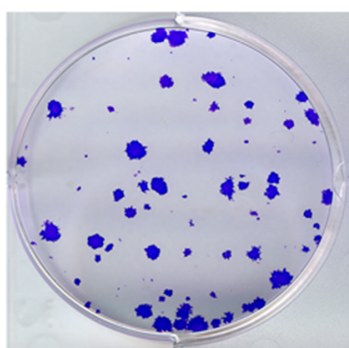

Ctrl-shRNA

Fig. 2 PF4V1 overexpression promoted cell viability and colony formation. The proliferation activity of the oral cells with PF4V1 stably transfected was detected by CCK-8 assay (a, b, $\mathbf{c}$ for SCC-9; $\mathbf{e}, \mathbf{f}, \mathbf{g}$ for CAL-27) at $24 \mathrm{~h}, 48 \mathrm{~h}$ and $72 \mathrm{~h}$. The representative pictures of SCC-9 $\mathbf{d}$ and CAL-27 $\mathbf{h}$ cells from plate colony formation assay. ${ }^{*} \mathrm{P}<0.05,{ }^{* * *} \mathrm{P}<0.01,{ }^{* * *} \mathrm{P}<0.001$ versus the corresponding control group. PF4V1: PF4V1 overexpression group; Ctrl-PF4V1: the control vector of PF4V1 overexpression group; Ctrl-shRNA: the control vector of PF4V1 silence group; PF4V1-shRNA: PF4V1 silence group 


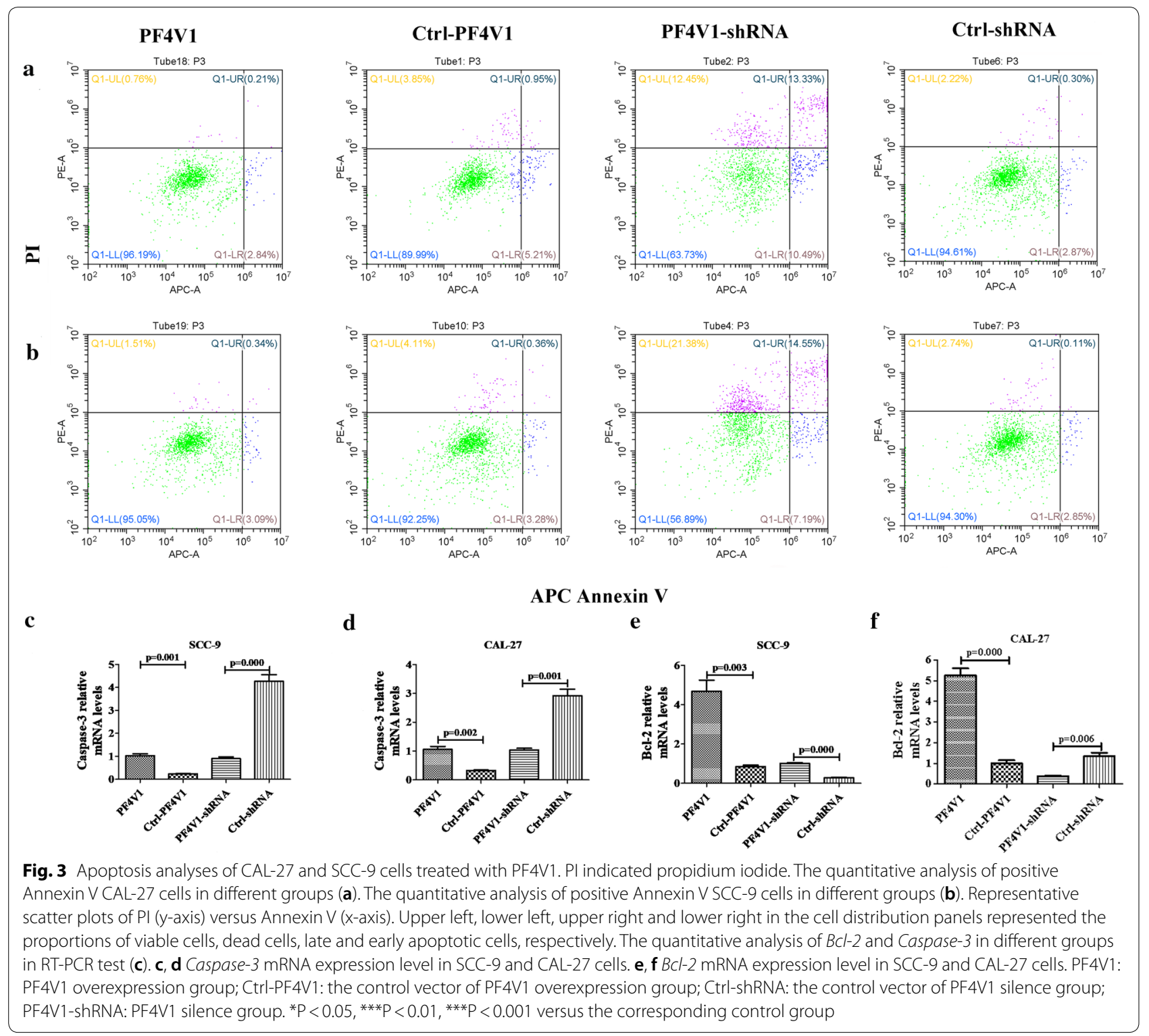

Our study found that PF4V1 knockdown led to the decreased expression levels of $\beta$-catenin, VEGF, FGF and the increased expression level of GSK-3 $\beta$ compared with those in the control groups. In addition, our study showed that PF4V1 knockdown or overexpression had no impact on the expression levels of snail1, snail2, twist compared with those in the control, as determined by RT-PCR and western blot analysis.

\section{Discussion}

Although great efforts have been made to find new treatments, cancer still remains a main threat against the health of mankind and a leading cause of death worldwide. The two main limitations of the current treatment methods are (1) the inability to stop the spread of cancer cells and (2) the difficulty in completing the primary tumor eradication. Compared with traditional treatments, the gene treatment of tumors in vivo has emerged as a promising and alternative therapy $[19,20]$. PF4V1 was evidenced to have statistically significant expressions in cancer tissue samples compared with para-carcinoma tissue samples [21, 22]. Additionally, our previous research also found that PF4V1 was not expressed or at a low level in the test samples from OSCC patients with lymph node metastasis compared with OSCC patients with no lymph node metastasis and the healthy population [18]. A close relationship existed between PF4V1 and OSCC. Moreover, the relationship between PF4V1 


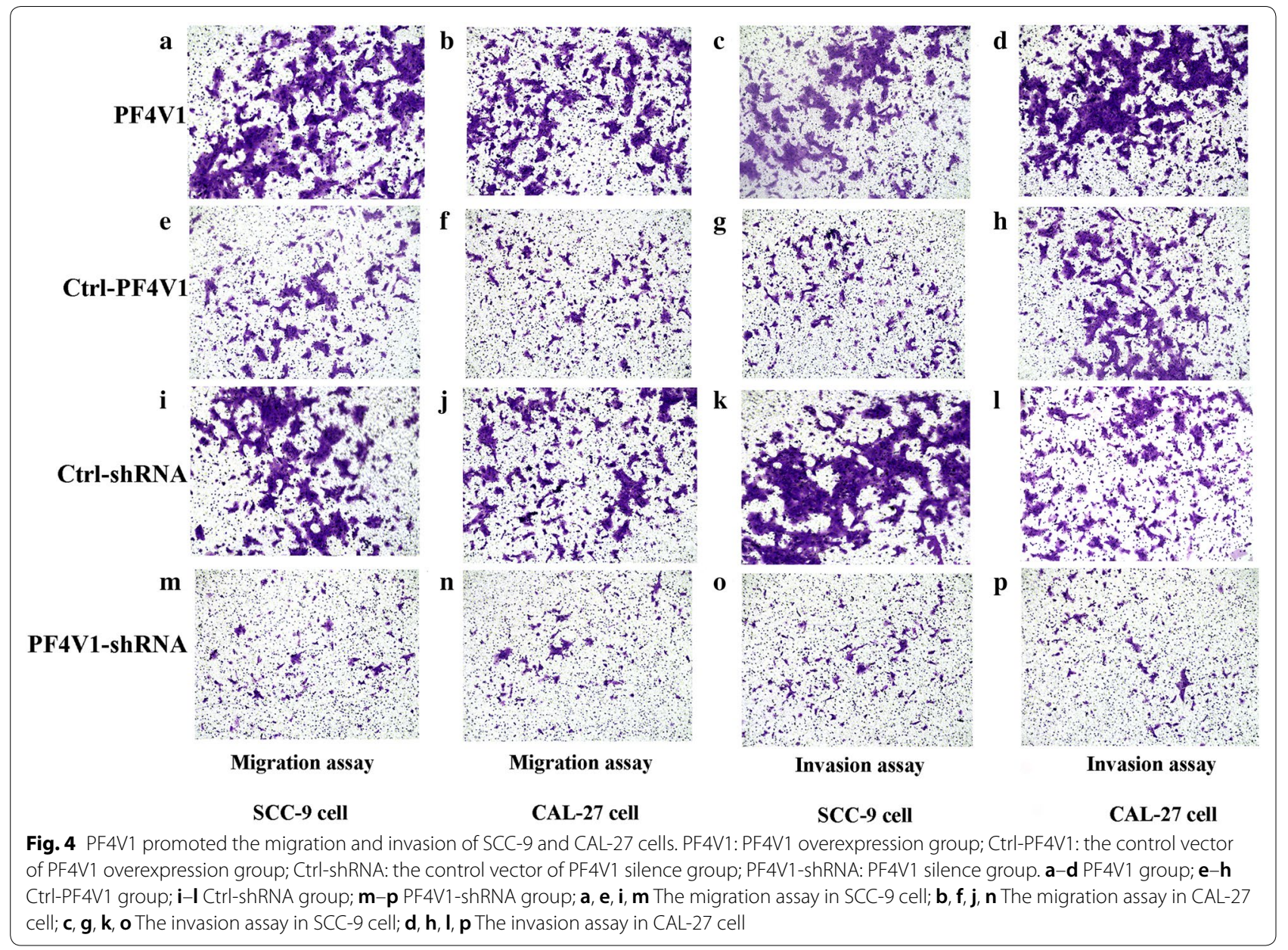

and the proliferation and tumor formation of OC cells was not yet completely understood. Therefore, this study explored the mechanism of the action of PF4V1 on OSCC to search for a potential anti-OSCC agent. It has been proved that application of angiostatin PF4V1 without attraction of protumoral phagocytes may be beneficial to the treatment of cancer [21].

Presently, PF4V1 had been found to have an inhibitory effect of vasculogenesis and affect the development and progression of carcinomas more directly. It has been demonstrated to be potent at impacting some biological behaviors of tumor cells, including proliferation, apoptosis, invasion, migration and angiogenesis [6, 23-25]. Our study showed that PF4V1 overexpression promoted proliferation, migration, and invasion in vitro and tumorigenesis of $\mathrm{OC}$ cells in vivo. An unlimited capability of angiogenesis was the hallmark of tumor cells. The roles of PF4V1 on tumor angiogenesis of multiple cancers had been reported previously [7]. Our study also showed that PF4V1 had an influence on VEGF and FGF expressions, exerting promotive effects on these angiogenic factors. In addition, the results of H\&E staining revealed that overexpression of PF4V1 led to active angiogenesis and low differentiation, indicating higher proliferation of tumor tissue. The present study investigated the precise molecular mechanism which was concerned in the promotion of OC cell proliferation mediated by PF4V1. However, some researchers had also reported that PF4V1 prevented carcinoma growth and metastasis by inhibition of angiogenesis $[7,26,27]$. This implied that the effect of PF4V1 on OC cells was very different from that in other tumors.

The evolutionarily conserved Wnt signaling played an important role in the development and growth of embryos and tissue homeostasis [28]. In addition, a recent survey proved that activated Wnt signaling was involved in the occurrence and progression of various tumors [29-31]. This study showed that PF4V1 overexpression and knockdown caused increase and decrease expressions of related proteins of the Wnt signaling pathway in OC cells, respectively. For example, as a key protein in the Wnt signaling pathway, the expression levels of $\beta$-catenin were also up-regulated in PF4V1 


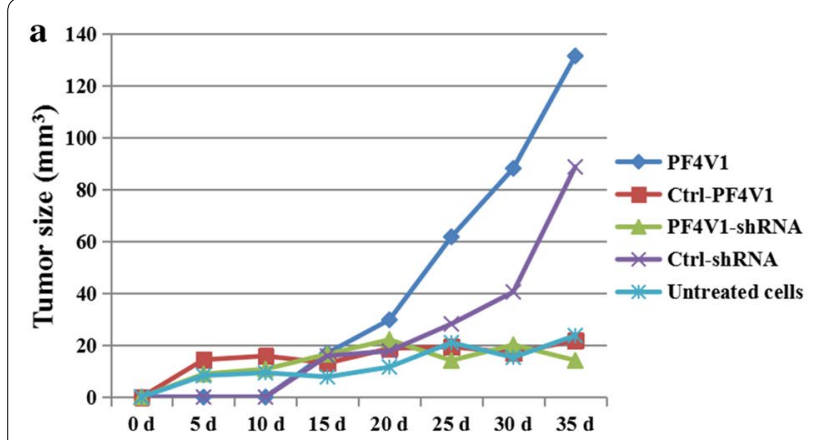

d

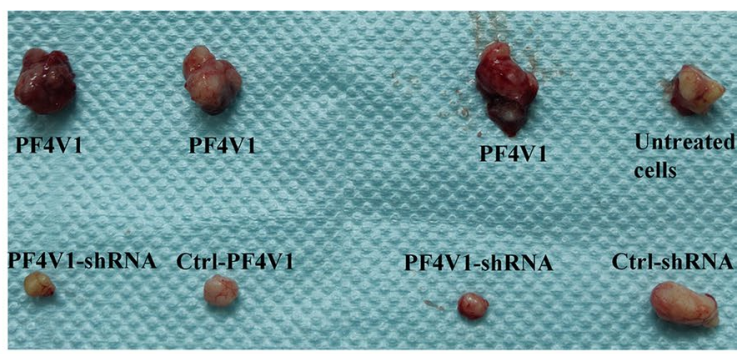

b

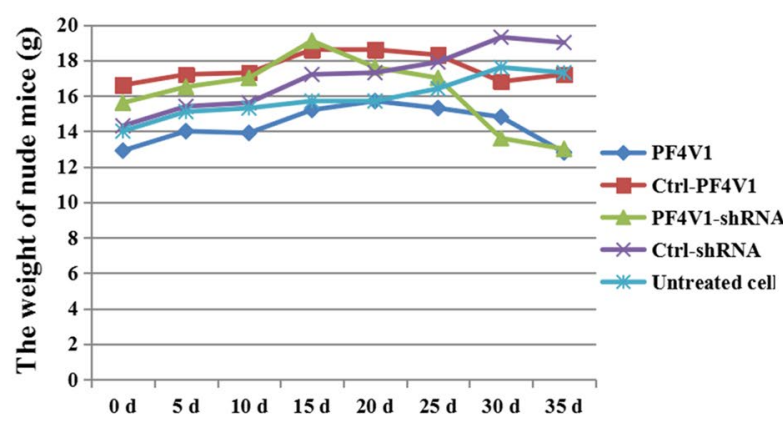

c

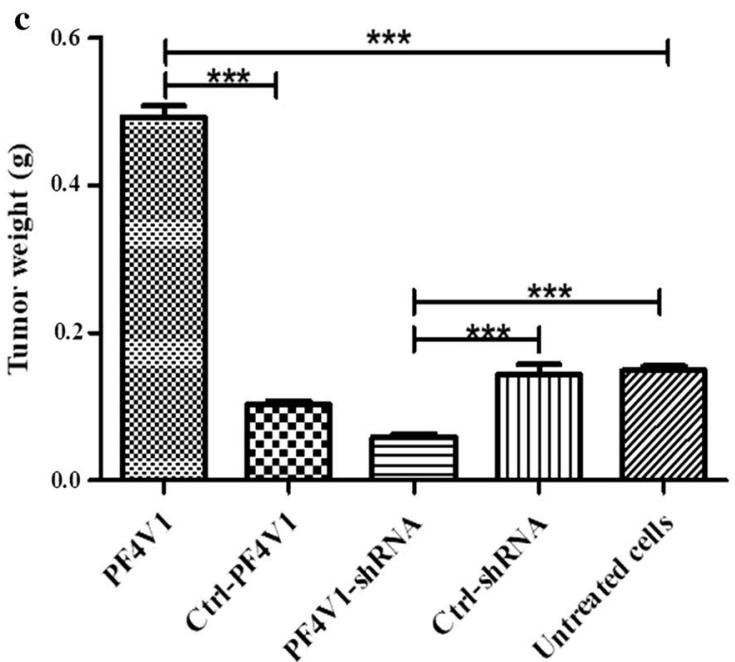

e
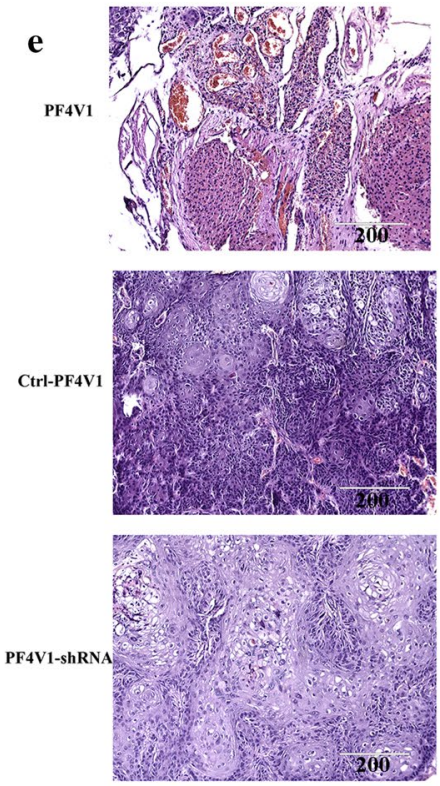

Ctrl-shRNA

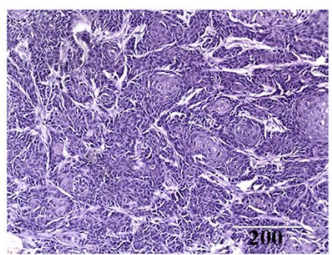

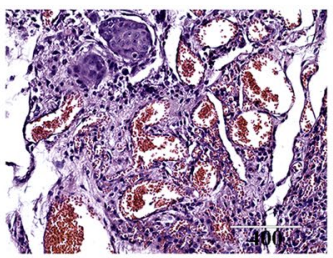
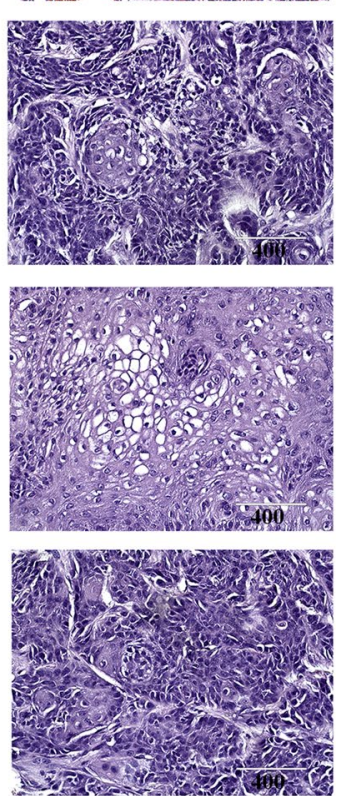

Fig. 5 PF4V1 affected the tumor formation of oral cancer cells in vivo. a The curves of tumor size were monitored every 5 days. $\mathbf{b}$ The curves of the weight of nude mice were monitored every 5 days. c Tumor weights were shown for overexpression and suppression expression of PF4V1 in SCC-9 and CAL-27 cells in the end of the study. ${ }^{*} \mathrm{P}<0.05,{ }^{* * *} \mathrm{P}<0.01,{ }^{* * *} \mathrm{P}<0.001$ versus the corresponding control group; $\mathbf{d}$ The tumors were separated from xenografts. e The HE staining results of tumor tissues and the pictures were enlarged 200 (mark 200 in the image) and 400 times (mark 400 in the image). PF4V1: PF4V1 overexpression group; Ctrl-PF4V1: the control vector of PF4V1 overexpression group; Ctrl-shRNA: the control vector of PF4V1 silence group; PF4V1-shRNA: PF4V1 silence group

overexpression cells and down-regulated in the knockdown cells. We also found that overexpression of PF4V1 significantly down-regulated the expression levels of
GSK-3 $\beta$. However, PF4V1 had no effect on EMT pathway. The PF4V1-regulated promotion of Wnt/ $\beta$-catenin signaling and the subsequent experiments on cellular 


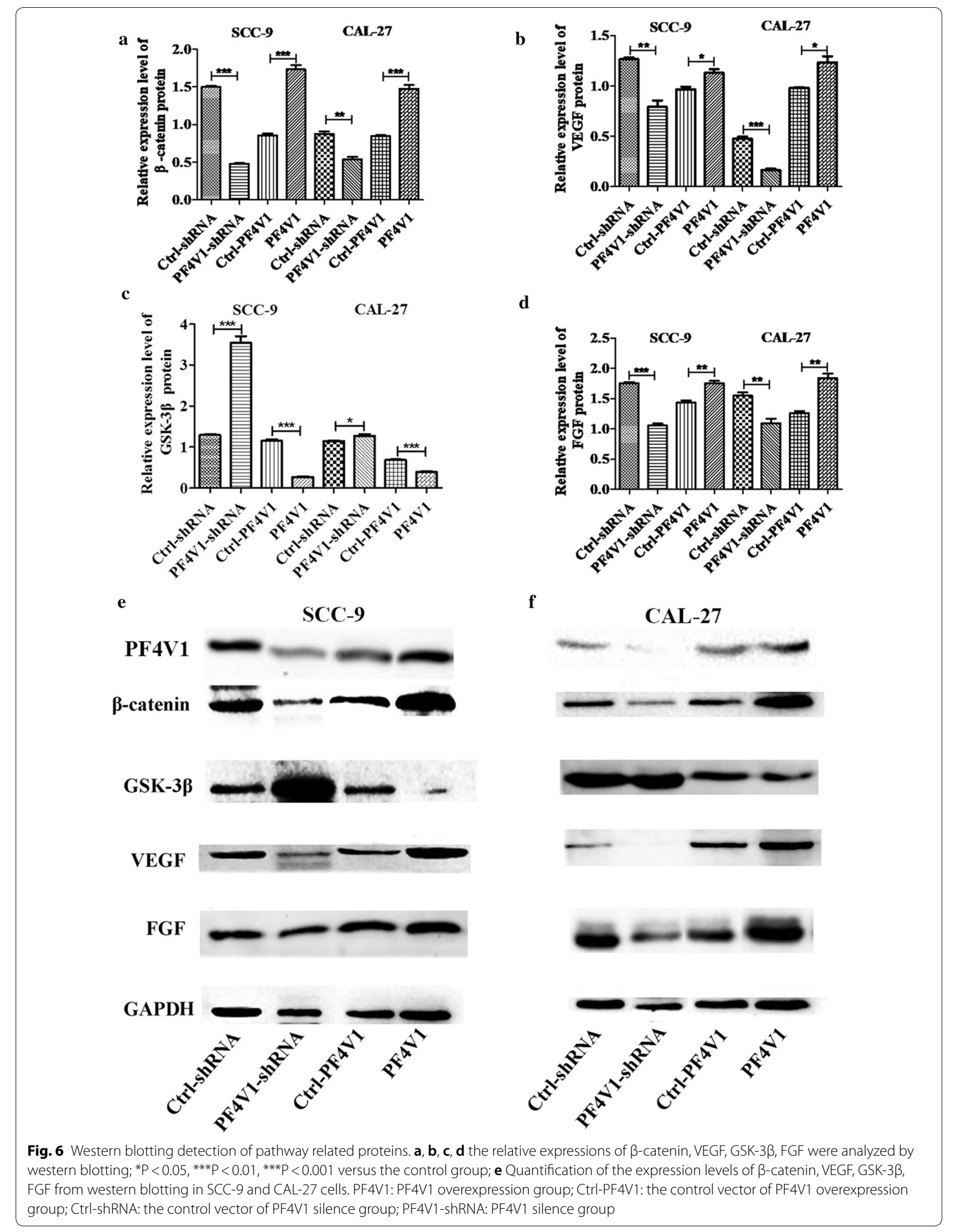




\section{function had not been reported in cancer. Our work sug- gested that PF4V1 did affect some biological behaviors of $\mathrm{OC}$ cells in vivo and in vitro by activating Wnt signaling.}

\author{
Acknowledgements
}

Not applicable.

\section{Authors' contributions}

The authors thank all of the study participants. All the participants conceived and designed the study; JWD and HXP conducted experimentation on animals; LCP and ZY conducted experiments in vitro; LCP and ZY carried out all statistical analysis; LCP, JWD and ZN completed the manuscript. All authors read and approved the final manuscript.

\section{Funding}

This work is supported by grants from Guangxi Natural Science Foundation (Grant Nos.:2018GXNSFAA138003), Youth Science Foundation of Guangxi Medical University (Grant Nos.:GXMUYSF201702), Guangxi scientific research and technology development program project (Grant Nos.: GGK0993003C-16), Guangxi Specially-invited Expert Special Foundation, Guangxi Specially-invited Expert Special Foundation, Guangxi Clinical Research Center for Craniofacial Deformity (Grant Nos.: GKAD17129004) and National Natural Science Foundation of China (Grant Nos.: 81670970, 81870748), Guangxi medical and health appropriate technology research and development project (Grant Nos: S201538). The funding body provided adequate funding in the design of the study and collection, analysis.

\section{Availability of data and materials}

The datasets used and/or analyzed during the current study are available from the corresponding author on reasonable request.

\section{Competing interests}

The authors declare that they have no competing interests.

\begin{abstract}
Author details
${ }^{1}$ Guangxi Key Laboratory of Oral and Maxillofacial Rehabilitation and Reconstruction, Guangxi Key Laboratory of Oral and Maxillofacial Surgery Disease Treatment, Guangxi Clinical Research Center for Craniofacial Deformity, School of Stomatology, Guangxi Medical University, Nanning 530021, People's Republic of China. ${ }^{2}$ Department of Oral and Maxillofacial Surgery, The Affiliated Stomatology Hospital of Guangxi Medical University, Nanning 530021, People's Republic of China. ${ }^{3}$ School of Information and Management, Guangxi Medical University, Nanning 530021, People's Republic of China.
\end{abstract}

Received: 6 November 2019 Accepted: 23 March 2020

Published online: 07 April 2020

\section{References}

1. Rosebush MS, Rao SK, Samant S, Gu W, Handorf CR, Pfeffer LM et al (2011) Oral cancer: enduring characteristics and emerging trends. J Tenn Dent Assoc 91(2):24-27

2. Warnakulasuriya S (2009) Global epidemiology of oral and oropharyngeal cancer. Oral Oncol 45(4-5):309-316

3. Kumar SR, Markusic DM, Biswas M, High KA, Herzog RW (2016) Clinical development of gene therapy: results and lessons from recent successes. Mol Ther Methods Clin Dev 3:16034

4. Negre $O$, Eggimann AV, Beuzard Y, Ribeil JA, Bourget $P$, Borwornpinyo $S$ et al (2016) Gene therapy of the beta-hemoglobinopathies by lentiviral transfer of the beta (A(T87Q))-globin gene. Hum Gene Ther 27(2):148-165

5. Johnson LA, Morgan RA, Dudley ME, Cassard L, Yang JC, Hughes MS et al (2009) Gene therapy with human and mouse T-cell receptors mediates cancer regression and targets normal tissues expressing cognate antigen. Blood 114(3):535-546

6. Struyf S, Burdick MD, Proost P, Van Damme J, Strieter RM (2004) Platelets release PF4L1, a nonallelic variant of the chemokine platelet factor-4/PF4 and potent inhibitor of angiogenesis. Circ Res 95(9):855-857
7. Struyf S, Burdick MD, Peeters E, Van den Broeck K, Dillen C et al (2007) Platelet factor-4 variant chemokine PF4L1 inhibits melanoma and lung carcinoma growth and metastasis by preventing angiogenesis. Cancer Res 67(12):5940-5948

8. Luo J, Yao Y, Ji S, Sun Q, Xu Y, Liu K et al (2019) PITX2 enhances progression of lung adenocarcinoma by transcriptionally regulating WNT3A and activating Wnt/beta-catenin signaling pathway. Cancer Cell Int 19:96

9. Xian-Li T, Hong L, Hong Z, Yuan L, Jun-Yong D, Peng X et al (2019) Higher expression of Linc00152 promotes bladder cancer proliferation and metastasis by activating the Wnt/ $\beta$-catenin signaling pathway. Med Sci Monit 25:3221-3230

10. Lyu Q, Jin L, Yang X, Zhang F (2019) LncRNA MINCR activates Wnt/ beta-catenin signals to promote cell proliferation and migration in oral squamous cell carcinoma. Pathol Res Pract 215(5):924-930

11. Acloque H, Adams MS, Fishwick K, Bronner-Fraser M, Nieto MA (2009) Epithelial-mesenchymal transitions: the importance of changing cell state in development and disease. J Clin Invest 119(6):1438-1449

12. Wei D, Wang W, Shen B, Zhou Y, Yang X, Lu G et al (2019) MicroRNA199a5p suppresses migration and invasion in oral squamous cell carcinoma through inhibiting the EMTrelated transcription factor SOX4. Int J Mol Med 44(1):185-195

13. Chen R, Jin G, Li W, McIntyre TM (2018) Epidermal growth factor (EGF) autocrine activation of human platelets promotes EGF receptordependent oral squamous cell carcinoma invasion, migration, and epithelial mesenchymal transition. J Immunol 201(7):2154-2164

14. Shi B, Ma C, Liu G, Guo Y (2019) MiR-106a directly targets LIMK1 to inhibit proliferation and EMT of oral carcinoma cells. Cell Mol Biol Lett 24:1

15. Dai J, Mu JW, Mu H (2019) Long non-coding RNA CRNDE regulates cell proliferation, migration, invasion, epithelial-mesenchymal transition and apoptosis in oral squamous cell carcinoma. Oncol Lett 17(3):3330-3340

16. Prats AC, Van den Berghe $L$, Rayssac A, Ainaoui N, Morfoisse F, Pujol F et al (2013) PF4L1-fibstatin cooperation inhibits tumor angiogenesis, lymphangiogenesis and metastasis. Microvasc Res 89:25-33

17. Vandercappellen J, Van Damme J, Struyf S (2011) The role of the CXC chemokines platelet factor-4 (PF4/PF-4) and its variant (PF4L1/PF-4var) in inflammation, angiogenesis and cancer. Cytokine Growth Factor Rev 22(1):1-18

18. Li CP, Zhou Y, Liu J, Su XP, Huang SH, Huang XP et al (2019) Potential markers from serum-purified exosomes for detecting oral squamous cell carcinoma metastasis. Cancer Epidemiol Biomarkers Prev 28(10):1668-1681

19. Breitbach CJ, Reid T, Burke J, Bell JC, Kirn DH (2010) Navigating the clinical development landscape for oncolytic viruses and other cancer therapeutics: no shortcuts on the road to approval. Cytokine Growth Factor Rev 21(2-3):85-89

20. Rommelaere J, Geletneky K, Angelova AL, Daeffler L, Dinsart C, Kiprianova I et al (2010) Oncolytic parvoviruses as cancer therapeutics. Cytokine Growth Factor Rev 21(2-3):185-195

21. Vandercappellen J, Noppen S, Verbeke H, Put W, Conings R, Gouwy M et al (2007) Stimulation of angiostatic platelet factor-4 variant (PF4L1/ PF-4var) versus inhibition of angiogenic granulocyte chemotactic protein-2 (CXCL6/GCP-2) in normal and tumoral mesenchymal cells. J Leukoc Biol 82(6):1519-1530

22. Verbeke H, De Hertogh G, Li S, Vandercappellen J, Noppen S, Schutyser E et al (2010) Expression of angiostatic platelet factor-4var/PF4L1 counterbalances angiogenic impulses of vascular endothelial growth factor, interleukin-8/CXCL8, and stromal cell-derived factor 1/CXCL12 in esophageal and colorectal cancer. Hum Pathol 41(7):990-1001

23. Dubrac A, Quemener C, Lacazette E, Lopez F, Zanibellato C, Wu WG et al (2010) Functional divergence between 2 chemokines is conferred by single amino acid change. Blood 116(22):4703-4711

24. Quemener C, Baud J, Boye K, Dubrac A, Billottet C, Soulet F et al (2016) Dual roles for PF4 chemokines and CXCR3 in angiogenesis and invasion of pancreatic cancer. Cancer Res 76(22):6507-6519

25. Struyf S, Salogni L, Burdick MD, Vandercappellen J, Gouwy M, Noppen $S$ et al (2011) Angiostatic and chemotactic activities of the CXC chemokine PF4L1 (platelet factor-4 variant) are mediated by CXCR3. Blood 117(2):480-488 
26. Van Raemdonck K, Berghmans N, Vanheule V, Bugatti A, Proost P, Opdenakker $\mathrm{G}$ et al (2014) Angiostatic, tumor inflammatory and anti-tumor effects of PF4(47-70) and PF4L1(47-70) in an EGF-dependent breast cancer model. Oncotarget 5(21):10916-10933

27. Vandercappellen J, Liekens S, Bronckaers A, Noppen S, Ronsse I, Dillen $\mathrm{C}$ et al (2010) The $\mathrm{COOH}$-terminal peptide of platelet factor-4 variant (PF4L1/PF-4var(47-70)) strongly inhibits angiogenesis and suppresses B16 melanoma growth in vivo. Mol Cancer Res 8(3):322-334

28. Krausova M, Korinek V (2014) Wnt signaling in adult intestinal stem cells and cancer. Cell Signal 26(3):570-579

29. Wang SH, Li N, Wei Y, Li QR, Yu ZP (2014) beta-catenin deacetylation is essential for WNT-induced proliferation of breast cancer cells. Mol Med Rep 9(3):973-978
30. Liu H, Yin J, Wang H, Jiang G, Deng M, Zhang G et al (2015) FOXO3a modulates WNT/beta-catenin signaling and suppresses epithelialto-mesenchymal transition in prostate cancer cells. Cell Signal 27(3):510-518

31. Ashihara E, Takada T, Maekawa T (2015) Targeting the canonical Wnt/ beta-catenin pathway in hematological malignancies. Cancer Sci 106(6):665-671

\section{Publisher's Note}

Springer Nature remains neutral with regard to jurisdictional claims in published maps and institutional affiliations.

\section{Submit your manuscript to a SpringerOpen ${ }^{\odot}$ journal and benefit from:}

- Convenient online submission

- Rigorous peer review

- Open access: articles freely available online

- High visibility within the field

- Retaining the copyright to your article

Submit your next manuscript at $\gg$ springeropen.com 